\title{
GERMINAÇÃO DE SEMENTES DE LAVOISIERA CORDATA COGN. E LAVOISIERA FRANCAVILLANA COGN. (MELASTOMATACEAE), ESPÉCIES SIMPÁTRICAS DA SERRA DO CIPÓ, BRASIL
}

\author{
Bernardo D. Ranieri ${ }^{1,2}$ \\ Tate C. Lana ${ }^{1}$ \\ Daniel Negreiros ${ }^{1}$ \\ Luzia M. Araújo ${ }^{1}$ \\ Geraldo Wilson Fernandes ${ }^{1,3}$
}

Recebido em 25/09/2002. Aceito em 08/05/2003

\begin{abstract}
RESUMO - (Germinação de sementes de Lavoisiera cordata Cogn. e Lavoisiera francavillana Cogn. (Melastomataceae), espécies simpátricas da Serra do Cipó, Brasil). Neste estudo verificou-se o comportamento germinativo de sementes de Lavoisiera cordata Cogn. e Lavoisiera francavillana Cogn. (Melastomataceae), espécies que ocorrem em hábitats com características hídricas e nutricionais diferentes nos campos rupestres da Serra do Cipó, Sudeste do Brasil. Sementes coletadas no ano de 2000 foram postas para germinar em quatro réplicas de 25 nas temperaturas de $15,20,25$ e $30^{\circ} \mathrm{C}$, sob fotoperíodo de $12 \mathrm{~h}$. A percentagem de germinação foi mais alta para $L$. cordata do que para $L$. francavillana $(\mathrm{t}=11.803, \mathrm{gl}=30, \mathrm{p}<0.01)$, assim como a velocidade de emergência de radícula $(\mathrm{t}=6.71, \mathrm{gl}=30, \mathrm{p}<0.01)$. L. cordata apresentou maiores velocidades de emergência (ANOVA, $\left.\mathrm{F}=14.282, \mathrm{r}^{2}=0.78, \mathrm{p}<0.01\right)$ a partir da temperatura de $20^{\circ} \mathrm{C}$, sendo que nessa mesma temperatura foi observada tendência a maiores percentagens de germinação ( $p>0.05$, N.S.). As percentagens de germinação correlacionaram-se positivamente com as velocidades de emergência para esta espécie $\left(\mathrm{r}^{2}=0.63, \mathrm{p}<0.05, \mathrm{y}=1.48 \mathrm{x}+1.97\right)$. L. francavillana apresentou as maiores taxas de germinação (ANOVA, $\mathrm{F}=3.388, \mathrm{r}^{2}=0.46, \mathrm{p}<0.05$ ) e tendência a maiores velocidades de emergência na temperatura de $25^{\circ} \mathrm{C}(\mathrm{p}>0.05$, N.S.), sendo que as percentagens de germinação se correlacionaram positivamente com as velocidades de emergência $\left(\mathrm{r}^{2}=0.90, \mathrm{p}<0,01, \mathrm{y}=4.32 \mathrm{x}-0.01\right)$. Os resultados indicam que as diferenças fisiológicas na germinação das sementes podem ser reflexo de adaptações às condições ecológicas às quais essas duas espécies estão sujeitas.
\end{abstract}

Palavras-chave - Lavoisiera, germinação de sementes, campos rupestres, temperatura, espécies ameaçadas

1 Ecologia Evolutiva de Herbívoros Tropicais, Departamento de Biologia Geral, ICB, Universidade Federal de Minas Gerais, C. Postal 486, CEP 30161-970, Belo Horizonte, MG, Brasil

2 Bolsista de Iniciação Científica da FAPEMIG

3 Autor para correspondência: gwilson@icb.ufmg.br 


\begin{abstract}
Seed germination of Lavoisiera cordata Cogn. and Lavoisiera francavillana Cogn. (Melastomataceae), sympatric plant species from Serra do Cipó, Brazil). In this study, the seed germination behavior of two sympatric plant species that occur in habitats under diferent nutritional and moisture conditions at Serra do Cipó, Southeastern Brazil, was evaluated. Seeds collected in the year of 2000 were placed to germinate in four replicates of 25 seeds under the temperatures at $15,20,25$ and $30^{\circ} \mathrm{C}$, and at 12 hour photoperiod. $L$. cordata showed higher germination percentage than $L$. francavillana $(\mathrm{t}=11.803, \mathrm{gl}=30, \mathrm{p}<0.01)$ as well as the radicle emergency rate $(\mathrm{t}=6.71, \mathrm{gl}=30, \mathrm{p}<0.01)$. L c cordata showed the highest radicle emergency rate (ANOVA, $\left.\mathrm{F}=14.282, \mathrm{r}^{2}=0.78, \mathrm{p}<0.01\right)$ under temperatures of $20^{\circ} \mathrm{C}$ and above. Under $20^{\circ} \mathrm{C}$ this species showed a tendency of higher germination percentages ( $p>0.05$, N.S.). The germination percentages were correlated with the the radicle emergency rate for this species $\left(\mathrm{r}^{2}=0.63, \mathrm{p}<0.05, \mathrm{y}=1.48 \mathrm{x}+1.97\right)$. L. francavillana showed the highest germination percentages (ANOVA, $\mathrm{F}=3.388, \mathrm{r}^{2}=0.46, \mathrm{p}<0.05$ ) and a tendency for higher the radicle emergency rate under $25^{\circ} \mathrm{C}\left(\mathrm{p}>0.05\right.$, N.S.). The germination percentages were also correlated with the radicle emergency rates $\left(r^{2}=0.90\right.$, $\mathrm{p}<0,01, \mathrm{y}=4.32 \mathrm{x}-0.01)$. Our results indicate fisiological diferences in the seed germination of $L$. cordata and $L$. francavillana. Those diferences may reflect adaptations to the diferent microhabitat conditions which the two species are subjected.
\end{abstract}

Key words - Lavoisiera, seed germination, rupestrian fields, temperature, threatened species

\section{Introdução}

Dentro do bioma Cerrado, a vegetação de campo rupestre representa uma das áreas mais críticas e ameaçadas (Lara \& Fernandes 1996; Costa et al. 1998; Ministério do Meio Ambiente 1999). Embora esta vegetação venha sendo estudada há mais de 100 anos (Warming 1892), quase nada se sabe sobre a biologia reprodutiva e propagação das suas espécies vegetais (Madeira \& Fernandes 1999; Ribeiro \& Fernandes 2000; Jacobi et al. 2000). Não obstante, é imperativo este conhecimento tendo em vista a necessidade premente para a recuperação ambiental dos campos rupestres, conservação e manejo de espécies nativas e/ou ameaçadas.

A utilização de espécies nativas na reabilitação ambiental não tem sido empregada devido à ausência de conhecimento consolidado sobre a biologia, ecologia, técnicas de propagação e manejo destas espécies. Por outro lado, a norma técnica 13.030 da ABNT (ABNT 1998), que regulamenta os projetos de reabilitação de áreas degradadas pela mineração, é clara no sentido da utilização de espécies nativas locais. Além disso, as espécies nativas apresentam interações nos processos ecológicos, mantendo a biodiversidade e a integridade biológica do ambiente (Karr 1991; 1993; Munn
1993; Steedman \& Haider 1993; Angermeier \& Karr 1997).

A Serra do Cipó está situada na porção Sul da Cadeia do Espinhaço, no Estado de Minas Gerais, Brasil, entre os paralelos $19^{\circ} 12^{\prime}$ $19^{\circ} 20^{\prime} \mathrm{S}$, à longitude de $43^{\circ} 30^{\prime}-43^{\circ} 40^{\prime} \mathrm{W}$, e apresenta-se sob forma de um conjunto de elevações em torno de $1.200 \mathrm{~m}$. A região é caracterizada por clima mesotérmico, com invernos secos de 4 a 5 meses e verões chuvosos de 7 a 8 meses, com média pluvial anual de $1.500 \mathrm{~mm}$ (Madeira \& Fernandes 1999). Seus solos são normalmente rasos, arenosos, secos e com pouca retenção de água (Giulietti et al. 1987; Ribeiro \& Fernandes 2000). No entanto, em campos brejosos da Serra do Cipó há alto teor de umidade devido ao fato dessas localidades estarem numa linha de drenagem, com solos constantemente saturados (Vitta 1995). Nesses campos brejosos o solo apresenta coloração escura devido ao alto teor de matéria orgânica, o que aumenta muito a capacidade de retenção de água nestes locais (Souza 1997).

A maioria das espécies de Lavoisiera (Melastomataceae) da Serra do Cipó ocorre tanto em áreas de campos brejosos e arenosos quanto em campos arenosos e pedregosos, apresentando caracteres morfológicos adaptados a cada tipo de ambiente (Souza 1997). O estudo deste gênero é muito importante devido à 
abundância e alta diversidade de espécies, e ainda à ampla associação com insetos herbívoros e polinizadores (B. Ranieri obs. pess.). Além disso, apresentam potencial ornamental devido tanto à arquitetura vegetativa, quanto às belas flores. Também relevante é o fato de várias espécies estarem ameaçadas ou em perigo de extinção (Mendonça \& Lins 2000).

Fatores físicos como disponibilidade hídrica do solo e temperatura promovem o aparecimento de características germinativas diversificadas (Vázquez-Yanes \& Orozco-Segovia 1996). O conhecimento da biologia de sementes de espécies filogeneticamente relacionadas mas que ocupam micro-hábitats distintos é importante para a compreensão de processos comunitários como sucessão, estabelecimento de plântulas e regeneração natural. O objetivo principal deste estudo foi conhecer o comportamento germinativo das sementes de duas espécies de Lavoisiera sob diferentes temperaturas. A espécie L. cordata Cogn. ocorre em hábitats xéricos, enquanto L. francavillana Cogn. habita áreas mésicas. Estas duas espécies foram selecionadas porque ocorrem em micro-hábitats com características muito distintas, apesar de serem espécies simpátricas e congenéricas. Este estudo fornece os primeiros dados que auxiliarão no conhecimento da biologia da germinação e reprodução destas espécies para subsidiar estudos futuros de reabilitação ambiental que utilizem plantas nativas de campos rupestres. Os dados obtidos serão também de valia para compreender os aspectos fisiológicos da germinação que refletem adaptações às circunstâncias ecológicas às quais as duas espécies estão sujeitas.

\section{Material e métodos}

Espécies estudadas - O gênero Lavoisiera engloba, principalmente, espécies de pequeno porte com flores geralmente isoladas, possuindo 6-8 pétalas de cores púrpura a branca. $\mathrm{O}$ ápice da antera é modificado em tubo e os estames são desiguais entre si (Barroso 1991). Há registro de 24 espécies de Lavoisiera na Serra do Cipó, distribuídas nas seções Gentianoidea, Muborosa, Cataphracta e Carinata (Souza 1997). São subarbustos ou arbustos que variam de cerca de 0,5-2,5m alt. com folhas opostas cruzadas e sésseis.

Lavoisiera francavillana Cogn. pertence à seção Cataphracta, possui flores roxo-claras e é comumente encontrada em grandes e densas manchas. Esta espécie tem como habitat campos brejosos sujeitos a encharcamento temporário por água proveniente das chuvas que caem com boa intensidade nos meses de outubro a março na Serra do Cipó (Souza 1997). Os órgãos vegetativos de L. francavillana apresentam adaptações anatômicas, como aerênquima nas raízes, que permitem que ela se estabeleça em solos encharcados (Souza 1997).

Lavoisiera cordata Cogn. pertence à seção Gentianoidea, apresenta flores brancas, ocorre isoladamente em campos pedregosos de melhor drenagem e, portanto, não possui aerênquima no sistema radicular (Souza 1997). Esta espécie foi recentemente classificada como ameaçada de extinção devido à área de distribuição restrita e ao pequeno tamanho de suas populações (Mendonça \& Lins 2000).

Ambas as espécies possuem porte arbustivo muito ramificado e denso, com florescimento vistoso de flores solitárias ou agrupadas com cerca de $6 \mathrm{~cm}$ diâm. na extremidade dos ramos e as estruturas foliares mostram principalmente características xeromórficas.

Coleta de frutos - Foram coletados aleatoriamente 500 frutos maduros de L. cordata e 500 de L. francavillana de populações localizadas na Reserva Particular Natural Vellozia, área adjacente ao parque Nacional da Serra do Cipó, MG (19¹7's, 4335' W), localizada entre os quilômetros 106 e 108 da rodovia MG 010 . Os frutos foram acondicionados em sacos de papel e secos à temperatura ambiente durante 48 horas. 
Teste de germinação - Sementes intactas e aparentemente viáveis foram selecionadas e colocadas em placas de Petri (quatro réplicas com 25 sementes) forradas com dupla camada de papel de filtro umedecido com solução de nistatina (500 U.I/1) (Gomes \& Fernandes 1994; Lemos Filho et al. 1997). As placas de Petri foram colocadas em câmaras de germinação sob fotoperíodo de 12 horas de luz branca fluorescente $\left(280 \mu \mathrm{mol} \mathrm{cm} \mathrm{cm}^{-2} \mathrm{seg}^{-1}\right)$ nas temperaturas constantes de $15^{\circ}, 20^{\circ}, 25^{\circ}$ e $30^{\circ} \mathrm{C}$ (Felippe \& Silva 1984; Cardoso 1997). Foi verificada a ocorrência de germinação a cada 24 horas, durante o período de 30 dias (Lieberg \& Joly 1993). A emergência da radícula através do tegumento da semente foi o critério de germinação utilizado (Lucas \& Arrigoni 1992).

Análise dos dados - As percentagens de germinação das sementes, de cada repetição, sob diferentes temperaturas foram submetidas à analise de variância (ANOVA) e suas médias comparadas pelo teste de Tukey (Cardoso 1997). Foi calculado o índice de velocidade de emergência da radícula (IVE), de acordo com a fórmula (Souza \& Varela 1998):

$\mathrm{IVE}=\mathrm{N} 1 / \mathrm{D} 1+\mathrm{N} 2 / \mathrm{D} 2+\ldots+\mathrm{Nn} / \mathrm{Dn}$ onde:

N1 = número de sementes germinadas no primeiro dia de contagem

$\mathrm{N} 2$ = número de sementes germinadas no segundo dia de contagem

$\mathrm{Nn}=$ número de sementes germinadas no enésimo dia de contagem

D1 = primeiro dia de contagem

D2 = segundo dia de contagem

Dn = enésimo dia de contagem

As percentagens de germinação foram tranformadas em arco-seno para normalização dos dados. Após a confirmação da distribuição normal destes dados com teste não paramétrico de Kolmogorov-Smirnov, foram realizados testes $t$ de Student para comparação entre espécies e análises de variância (ANOVA) para avaliar, dentro de cada espécie, as diferenças em cada temperatura (Zar 1984). Após calculados o IVE de cada repetição, os dados foram submetidos à análise de variância para comparar as temperaturas, e a testes $t$ de Student para comparar as espécies (Zar 1984). Para verificar a relação entre as percentagens de germinação e os IVEs, foi utilizada a análise de regressão (Zar 1984). Todas as análises foram realizadas utilizando-se o programa SYSTAT 8.0 for Windows.

\section{Resultados}

Lavoisiera cordata apresentou maiores percentagens de germinação $(\mathrm{t}=11.803, \mathrm{gl}=30$, $\mathrm{p}<0.01)$ e maiores velocidades de emergência das sementes $(\mathrm{t}=6.71, \mathrm{gl}=30, \mathrm{p}<0.01)$ do que Lavoisiera francavillana (Fig. 1 e 2).

L. cordata apresentou maiores velocidades de emergência $\left(\mathrm{F}=14.282, \mathrm{r}^{2}=0.78, \mathrm{p}<0.01\right)$ nas temperaturas acima de $15^{\circ} \mathrm{C}$, sendo que a velocidade de emergência se mostrou estatisticamente superior a $20^{\circ} \mathrm{C}$. Foi observada apenas tendência a maiores percentagens de germinação a $20^{\circ} \mathrm{C}$ do que nas outras temperaturas ( $p>0.05$, N.S), sendo que estas foram relativa-

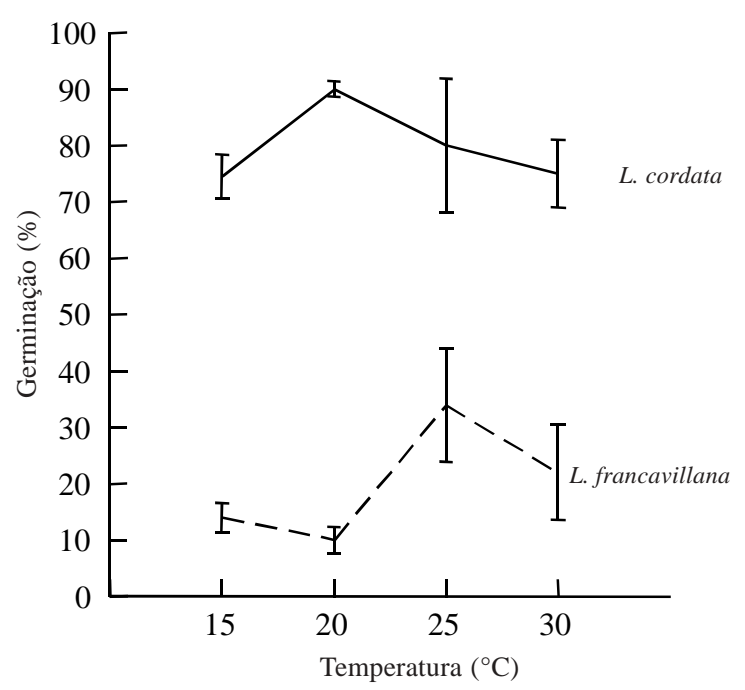

Figura 1. Percentagens médias de germinação de L. cordata e L. francavillana em relação ao gradiente de temperatura de 15 a $30^{\circ} \mathrm{C}$. 


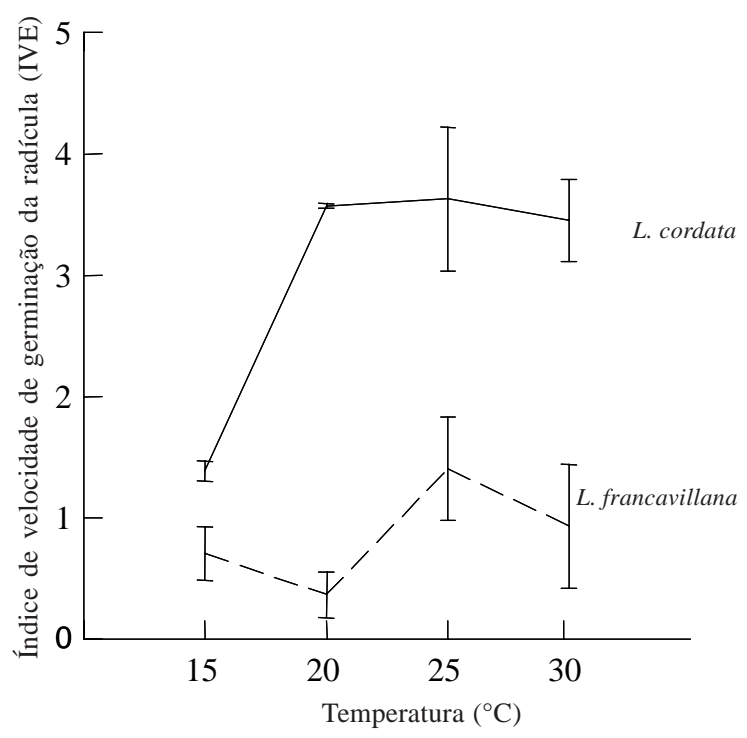

Figura 2. Velocidades médias de emergência de radículas de $L$. cordata e $L$. francavillana em relação ao gradiente de temperatura de 15 a $30^{\circ} \mathrm{C}$.

mente altas em todas as temperaturas exceto à temperatura de $15^{\circ} \mathrm{C}$ (Fig. 1 e 2). A faixa de temperatura entre 15 e $30^{\circ} \mathrm{C}$ mostrou-se favorável à germinação de L. cordata, com percentagens acima de 60\% (Fig. 1). As percentagens de germinação correlacionaram-se positivamente com as velocidades de emergência para esta espécie $\left(r^{2}=0.63, p<0.05\right.$, $y=1.48 x+1.97)$ (Fig. 3).

O comportamento germinativo das sementes de L. francavillana foi muito diferente daquele de sementes de L. cordata. Esta espécie apresentou as maiores percentagens de germinação $\left(\mathrm{F}=3.388, \mathrm{r}^{2}=0.46, \mathrm{p}<0,05\right)$ e tendência a maiores velocidades de emergência na temperatura de $25^{\circ} \mathrm{C}$ ( $\mathrm{p}>0.05$, N.S.) do que nas outras temperaturas (Fig. 1 e 2). As taxas de germinação e velocidade de emergência de L. francavillana foram baixas em todas as temperaturas quando comparadas às de L. cordata (Fig. 1 e 2). Todavia, estas foram significativamente superiores na temperatura de $25^{\circ} \mathrm{C}$. As percentagens de germinação também correlacionaram-se positivamente com as velocidades de emergência para $L$. francavillana $\left(\mathrm{r}^{2}=0.90, \mathrm{p}<0.01, \mathrm{y}=4.32 \mathrm{x}-0.01\right)$ (Fig. 3). No

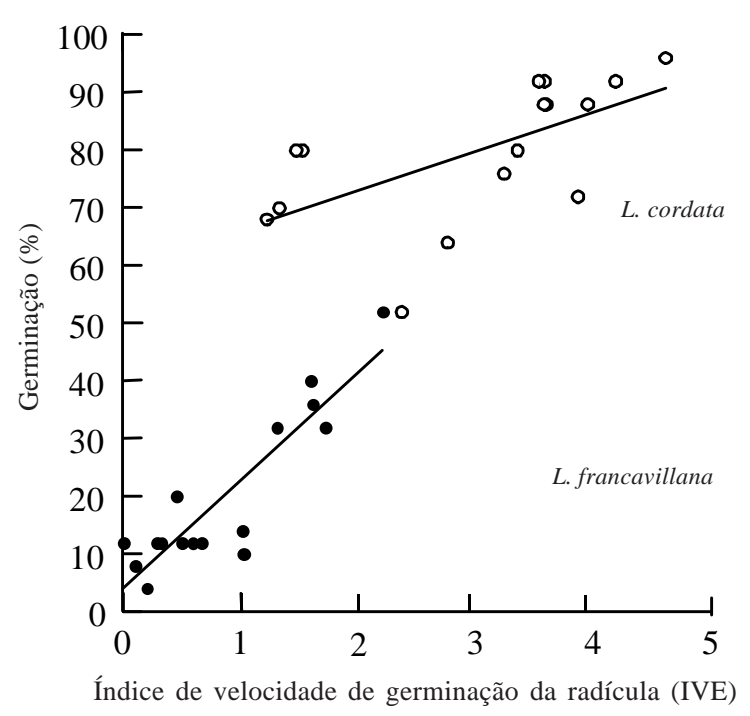

Figura 3. Correlação positiva entre a taxa de germinação e velocidade de emergência de radícula de $L$. cordata $(\bigcirc)$ e L. francavillana $(\bullet)$.

entanto, a reta resultante de tal correlação foi mais inclinada para esta espécie, indicando maior variação no aumento de taxas de germinação com menor variação do aumento de velocidade de emergência.

\section{Discussão}

O crescimento de plantas não é normalmente restringido pela disponibilidade de água do começo da estação úmida até 20-30 dias depois das chuvas (Fitter \& Hay 1987). Após este período, toda a água disponível nos primeiros 1-2 metros do solo desaparece progressivamente pela evapotranspiração. Sementes dispersadas no solo seco, no final da estação úmida, podem experimentar condições favoráveis para a germinação durante períodos isolados de chuva; no entanto, rapidamente não haverá mais água disponível para as plântulas resultantes (Fitter \& Hay 1987).

Plantas que crescem onde a água é disponível durante apenas parte do ano, como no campo rupestre da Serra do Cipó, devem ser capazes de fazer bom uso deste recurso durante o período favorável para o crescimento 
vegetativo. Como consequiência, as sementes de espécies de hábitats secos estão normalmente prontas para a dispersão no final ou depois da estação úmida, quando as condições são favoráveis para a germinação. Portanto, as sementes de muitas destas espécies podem estar dormentes logo que lançadas pela planta-mãe, e não germinarão até o começo de período prolongado de condições favoráveis (Fitter \& Hay 1987; Madeira \& Fernandes 1999).

Apesar de pertencerem ao mesmo gênero e serem espécies simpátricas, L. cordata e L. francavillana apresentaram comportamentos germinativos diferentes. Todavia, as duas espécies possuem, em comum, temperaturas ótimas de germinação entre 20 e $25^{\circ} \mathrm{C}$, temperaturas representativas dos meses mais quentes e úmidos nas regiões de Cerrado.

A menor velocidade de emergência apresentada pelas sementes de L. francavillana pode ser reflexo de sua adaptabilidade ao seu hábitat, representado por ambientes sujeitos a encharcamento temporário por água proveniente das chuvas (Souza 1997). Altas taxas de germinação das sementes desta espécie, precedida pela reidratação das mesmas, somente poderiam ocorrer após longo período de imersão na água, o que indicaria a estação chuvosa. Período mais longo de embebição das sementes de L. francavillana parece ser necessário para que a germinação ocorra em ambiente úmido e estável o suficiente para o sucesso do estabelecimento das plântulas resultantes. É possível que as amostras apresentassem sementes em dormência, que estariam aptas a germinar apenas no próximo período de condições favoráveis, uma vez que foram observadas baixas percentagens de germinação em todas as temperaturas. A maior variação do aumento da taxa de germinação relativo à variação do aumento da velocidade de emergência, representada pela inclinação da reta de regressão linear (Fig. 3), indica que L. francavillana apresentou relativamente maiores percentagens de germinação em amostras onde houve menor velocidade de emergência, em comparação com L. cordata. L. francavillana atingiu as mais altas percentagens e velocidades de germinação na temperatura de $25^{\circ} \mathrm{C}$, indicando que o mês de novembro (já na estação chuvosa), quando a temperatura média atinge este patamar (Madeira \& Fernandes 1999), pode ser o período mais favorável para a germinação das suas sementes. Este período de chuvas mais intensas seria responsável pela formação da lâmina de água observada no hábitat de $L$. francavillana e que poderia ter enorme importância no estabelecimento das plântulas desta espécie.

Lavoisiera cordata apresentou maiores percentagens de germinação e maiores velocidades de emergência do que $L$. francavillana em todas as temperaturas testadas. Tal resultado indica que esta espécie não tem necessidade de tempo tão longo de embebição para germinar como L. francavillana, pois, em seu hábitat, a água não fica acumulada na camada superficial do solo. A temperatura de $20^{\circ} \mathrm{C}$ pode ser a indicada como ótima para a germinação de L. cordata, pois esta foi a temperatura na qual esta espécie, além de atingir a mais alta percentagem de germinação, apresentou maior velocidade de emergência de radícula. Tal fato indica que as sementes desta espécie podem estar adaptadas a germinar nos meses que precedem a estação chuvosa (setembro e outubro), durante os quais a temperatura média oscila em torno de $20^{\circ} \mathrm{C}$ (Madeira \& Fernandes 1999).

As diferenças adaptativas na germinação de L. cordata e L. francavillana indicam que, quanto à germinação, estas duas espécies parecem ser bem adaptadas aos hábitats onde ocorrem e, juntamente com outras espécies de Lavoisiera, ajudam a caracterizar fisionomicamente os campos rupestres.

\section{Agradecimentos}

Os autores agradecem Celice Alexandre Silva, pela ajuda na coleta de dados; a José 
Rubens Pirani, pela identificação das espécies; a Vânia Gomes, pelo auxílio em todas as etapas deste trabalho; a Queila Garcia e José Pires de Lemos-Filho, pelas sugestões na versão inicial do manuscrito; aos revisores, pelas valiosas sugestões na versão final; à FAPEMIG (388/01), pela concessão de bolsa de Iniciação Científica ao primeiro autor; ao CNPq (469684/2001-4); à Idea Wild e Planta Tecnologia Ambiental, pelo apoio logístico.

\section{Referências bibliográficas}

ABNT - Associação Brasileira de Normas Técnicas 1998. Elaboração e apresentação de projetos de reabilitação de áreas degradadas pela mineração. NBR 13030, Brasil.

Angermeier, P. L. \& Karr, J. R. 1997. Biological integrity versus biological diversity as policy directives: protecting biotic resources. Pp. 264-275. In: F. B. Samson \& F. L. Knopf (eds.). Ecosystem management: selected readings. Springer, N.Y.

Barroso, G. M. 1991. Sistemática de Angiospermas do Brasil. Imprensa Universitária, Viçosa.

Cardoso, V. J. M. 1997. Germinação e desenvolvimento inicial de algumas espécies invasoras de culturas em diferentes tipos de solo. Naturalia 22: 61-74.

Costa, C. M. R.; Hermann, G. C. S.; Martins, L. V. Lins \& Lamas, I. R. 1998. Biodiversidade em Minas Gerais. Fundação Biodiversitas, Belo Horizonte.

Fitter, A. H. \& Hay R. K. 1987. Environmental physiology of plants. $2^{\text {nd }}$ ed. Academic Press, London.

Felipe, G. M. \& Silva, J. C. S. 1984. Estudos de germinação em espécies de cerrado. Revista Brasileira de Botânica 7: 157-163.

Giulietti, A. M.; Menezes, N. L.; Pirani, J. R.; Meguro, M. \& Wanderley, M. G. L. 1987. Flora da Serra do Cipó, Minas Gerais: caracterização e lista das espécies. Boletim de Botânica 9: 1-153.

Gomes, A. L. \& Fernandes, G. W. 1994. Influence of the parasitism of Cilostyles ingae (Rafflesiaceae) on its host plant, Mimosa naguirei (Leguminosae). Annals of Botany 74: 205-208.

Jacobi, C. M.; Carmo, R. M. \& Oliveira, R. S. 2000. The reproductive biology of two species of Diplusodon Pohl (Lithraceae) from Serra do Cipó, southeastern Brazil. Plant Biology 2: 670-676.
Karr, J. R. 1991. Biotic integrity: a long-neglected aspect of water resource management. Ecological Applications 1: 66-84.

Karr, J. R. 1993. Measuring biological integrity. Pp. 83-104. In: S. Woodley; J. Kay \& G. Francis (eds.). Ecological integrity and the management of ecosystems. St. Lucie Press, Ottawa.

Lara, A. C. F. \& Fernandes, G. W. 1996. The highest diversity of galling insects: Serra do Cipó, Brazil. Biodiversity Letters 3: 111-114.

Lemos Filho, J. P.; Guerra, S. T. M.; Lovato, M. B. \& Scotti, M. R. M. M. L. 1997. Germinação de sementes de Senna macranthera, Senna multifuga e Stryphnodendron polyphyllum. Pesquisa Agropecuária Brasileira 32: 357-361.

Lieberg, S. A. \& Joly, C. A. 1993. Inga affinis DC (Mimosaceae): germinação e tolerancia de plântulas à submersão. Revista Brasileira de Botânica 16: 175-179.

Lucas, N. M. C. \& Arrigoni, M. F. 1992. Germinação de sementes de Canavallia rosea ( $\mathrm{Sw}$ ) DC (Fabaceae). Revista Brasileira de Botânica 15: 105-112.

Madeira, J. A. \& Fernandes, G. W. 1999. Reproductive phenology of sympatric species of Chamaecrista (Leguminosae) in Serra do Cipó, Brazil. Journal of Tropical Ecology 15: 463-479.

Mendonça, M. P. \& Lins, L. V. 2000. Livro Vermelho das espécies ameaçadas de extinção da flora de Minas Gerais. Belo Horizonte: Fundação Biodiversitas, Fundação Zôo Botânica de Belo Horizonte.

Ministério do Meio Ambiente - MMA. 1999. Ações prioritárias para a conservação da biodiversidade do cerrado e pantanal. Ministério do Meio Ambiente, Funatura, Conservation International, Fundação Biodiversitas e Universidade de Brasília, Brasília.

Munn, R. E. 1993. Monitoring for ecosystem integrity. Pp. 105-116. In: S. Woodley; J. Kay \& G. Francis (eds.). Ecological integrity and the management of ecosystems. St. Lucie Press, Ottawa.

Ribeiro, K. T. \& Fernandes, G. W. 2000 Patterns of abundance of a narrow endemic species in a tropical and infertile habitat. Plant Ecology 147: 205-218.

Schütz, W. \& Milberg, P. 1997. Seed dormancy in Carex canascens: regional differences and ecological consequences. Oikos 78: 420-428. 
Souza, H. C. 1997. Estudo comparativo de adaptações anatômicas em órgãos vegetativos de espécies de Lavoisiera D.C. (Melastomataceae) da Serra do Cipó, MG. Tese de Doutorado. Universidade Estadual de Campinas, Campinas.

Souza, S. G. A. \& Varela, V. P. 1998. Tratamentos prégerminativos em sementes de faveira orelha de macaco (Enterolobium schomburgkii, Benth.). Acta Amazônica 19: 19-26

Steedman, R. \& Haider, W. 1993. Applying notions of ecological integrity. Pp. 47- 60. In: S. Woodley; J. Kay \& G. Francis (eds.). Ecological integrity and the management of ecosystems. St. Lucie Press, Ottawa.
Vasquez-Yanes, C. \& Orozco-Segovia, A. 1996. Patterns of seed longevity and germination in the tropical rain forest. Annual Review of Ecology and Systematics 24: 67- 87.

Vitta, F. A. 1995. Composição florística e ecologia de comunidades campestres na Serra do Cipó, Minas Gerais. Dissertação de Mestrado, Universidade de São Paulo, São Paulo.

Warming, E. 1892. Lagoa Santa. Et Bidrag til den biologiske. Planteografi. K. danske videns K. Selsk. Skr 6- Copenhague.

Zar, J. H. 1984. Biostatistical Analisys, $2^{\text {nd }}$ ed. Prentice-Hall, Englewood Ciffs, N. J. 